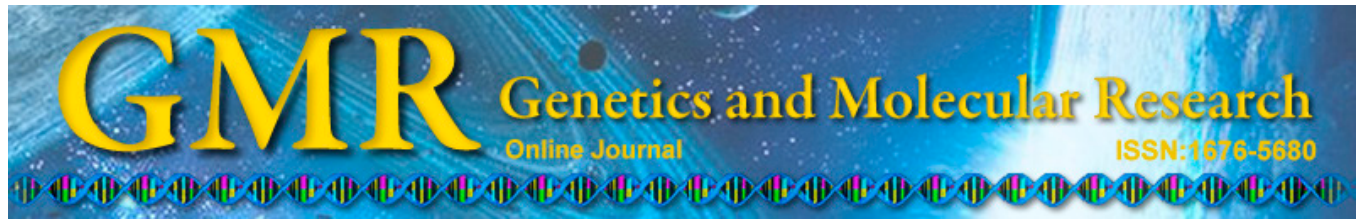

\title{
Identification of a novel duplication mutation in the $V H L$ gene in a large Chinese family with Von Hippel-Lindau (VHL) syndrome
}

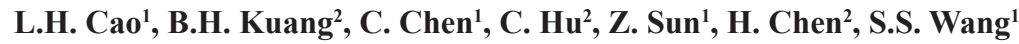 \\ and Y. Luo ${ }^{1}$ \\ ${ }^{1}$ Key Laboratory of Medical Cell Biology, \\ The Research Center for Medical Genomics, Ministry of Education, \\ China Medical University, Shenyang, Liaoning, China \\ ${ }^{2}$ Department of Genetic Research, Medical College of Nanchang University, \\ Nanchang, Jiangxi, China \\ Corresponding author: B.H. Kuang \\ E-mail: bhkuang@aliyun.com \\ Genet. Mol. Res. 13 (4): 10177-10183 (2014) \\ Received January 3, 2014 \\ Accepted April 15, 2014 \\ Published December 4, 2014 \\ DOI http://dx.doi.org/10.4238/2014.December.4.12
}

\begin{abstract}
Von Hippel-Lindau (VHL) syndrome is characterized by hemangioblastomas of the brain, spinal cord, and retina, renal cysts, clear cell renal cell carcinoma, and pheochromocytoma. VHL is caused by mutations in the VHL tumor suppressor gene. We attempted to detect mutation in the $V H L$ gene in a 5-generation Chinese family with VHL. We identified a novel small duplication that altered the reading frame downstream and created a premature TGA stop signal, resulting in severely truncated pVHL30 (p.Gly114Serfs*50) and pVHL19 (p.Gly61Serfs*50). This change was predicted to be an elongin-binding domain deletion.
\end{abstract}

Key words: Hemangioblastoma; Von Hippel-Lindau disease; Duplication mutation 


\section{INTRODUCTION}

Von Hippel-Lindau disease (VHL, MIM\#193300) is a rare, autosomal dominant, inherited disorder characterized by predisposition to central nervous system hemangioblastoma, retinal hemangioma, renal cysts, and clear cell renal cell carcinoma (RCC), pheochromocytoma, pancreatic cysts and neuroendocrine tumors, endolymphatic sac tumors, epididymal, and broad ligament cysts (Kaelin and Maher, 1998). Hemangioblastomas and RCC are the 2 most common manifestations of VHL disease, occurring in up to $70-80 \%$ patients. The incidence of VHL disease is approximately 1 in 36,000 in the general population, with an overall penetrance of $>90 \%$ by 65 years (Maher et al., 1991).

Based upon clinical features of the disease, VHL disease has been divided into 2 main types based on the absence (type 1) or presence (type 2) of pheochromocytoma. Type 1 is characterized by retinal and central nervous system hemangioblastoma, renal cell carcinoma, and a low risk for pheochromocytoma, while type 2 is characterized by a high risk for pheochromocytoma. Type 2 is further subdivided into type $2 \mathrm{~A}$ (pheochromocytoma without RCC or pancreatic cysts), 2B (pheochromocytoma with RCC or pancreatic cysts), and 2C (only pheochromocytoma) (Hes et al., 2005).

VHL tumor suppressor gene ( $V H L)$ is the only known gene to be associated with VHL syndrome, and mutations responsible for the disease have been identified in nearly $100 \%$ of affected families (Wong et al., 2007). Approximately $80 \%$ of individuals diagnosed with VHL disease inherited the disorder from a family member, while $20 \%$ of cases are caused by a de novo VHL mutation (Maher et al., 2011). VHL is located on 3p25-26 and its coding sequence is present in 3 exons that encode 2 VHL proteins: a full length 213-amino acid protein (pVHL30) and a smaller protein (pVHL19) that lacks the first 53 amino acids, both of which bind to elongin $\mathrm{B}$ and $\mathrm{C}$ to inhibit the activity of the elongin complex to decrease premature transcriptional termination. Functional studies suggest that the two pVHL isoforms have equivalent effects (Schoenfeld et al., 1998; Stebbins et al., 1999).

In this study, we identified a novel 14-base pair duplication in a large Chinese family with VHL disease. Identification of the disease-causative mutation was found to be consistent with the clinical diagnosis of VHL disease. Our results provide information that can be used in the genetic counseling of other family members.

\section{MATERIAL AND METHODS}

\section{Patients}

A family with VHL (Figure 1) included more than 70 at-risk individuals over 5 generations. Symptomatic individuals received magnetic resonance imaging scans of the head and spinal cord, abdominal computed tomography scans, and examination of the retina by an ophthalmologist.

\section{Mutation analysis}

Blood samples were collected from 35 members ( 5 confirmed affected individuals and 1 spouse) after obtaining informed consent and approval from the Nanchang University Institutional Review Board. Genomic DNA was extracted from peripheral blood using the standard 
sodium dodecyl sulfate-proteinase K-phenol/chloroform method. The proband was screened for mutations in the VHL gene, including genomic DNA fragments corresponding to each exon and their flanking intronic sequences. They were amplified by polymerase chain reaction (PCR) and subjected to automatic DNA sequencing after purification. The affected allele was determined by cloning the PCR amplicon into the $\mathrm{pMD}^{\mathrm{TM}} 18-\mathrm{T}$ vector (TaKaRa; Shiga, Japan) and then sequencing using the primers RV-M and M13-47. To confirm the pathogenicity of the variation, the amplicons of exon 1 from 34 additional family members and 70 normal controls were sequenced. Moreover, we performed 2 real-time quantitative PCR assays to determine the relative copy number of $V H L$.

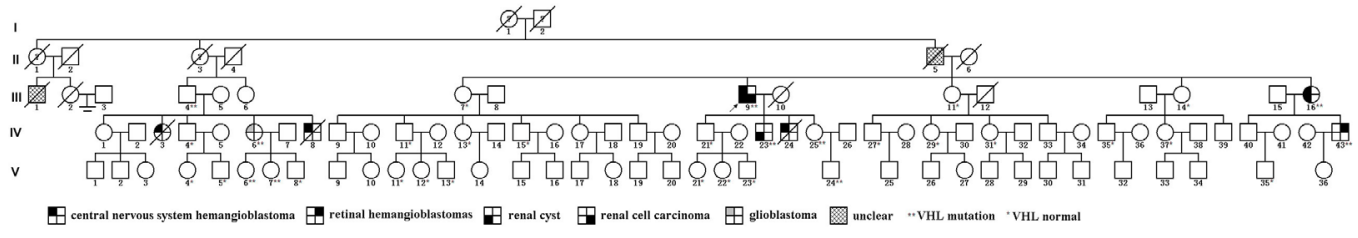

Figure 1. Pedigree of the family with VHL disease. The arrow indicates the proband.

\section{RESULTS}

\section{Clinical study}

Eight affected individuals were retrospectively identified by reviewing medical records and from the recall of relatives. Three had passed away before 40 years of age because of central nervous system hemangioblastoma. Among the 5 living patients, 3 had renal cysts (Figure 2) and 1 had developed renal cell carcinoma, 2 had central nervous system hemangioblastoma, 1 had retinal hemangioblastomas, and 1 was diagnosed with glioblastoma (Table 1).

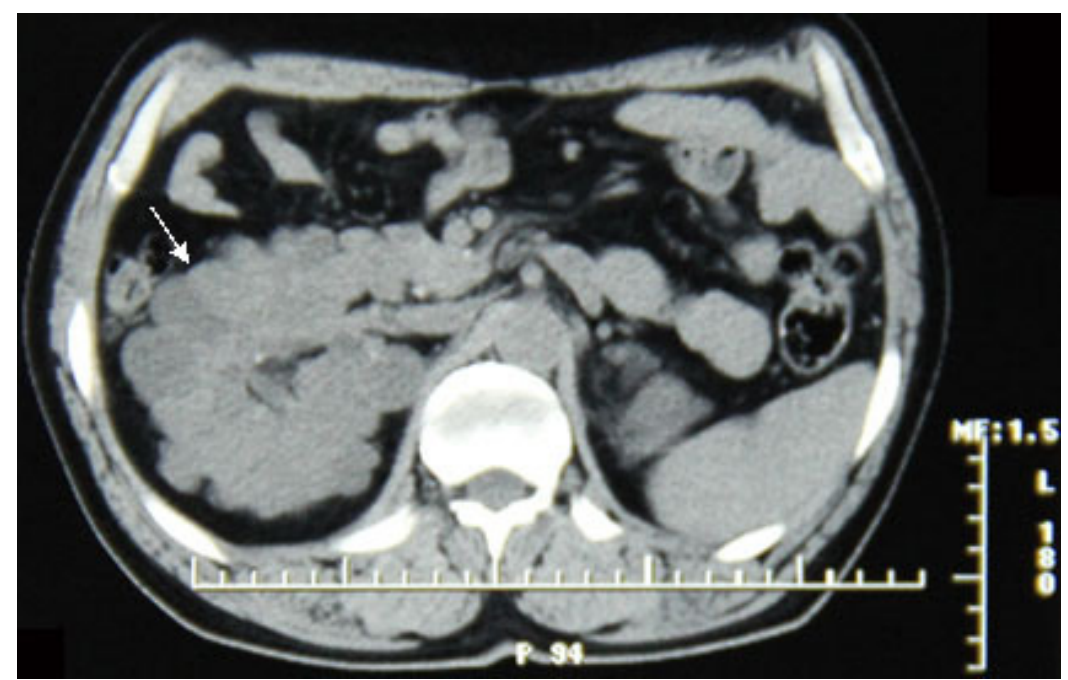

Figure 2. Abdominal computed tomography scanning image of the proband. The arrow indicates multiple renal cysts. 
Table 1. Clinical information and genotypes of individuals from the Chinese family with VHL syndrome.

\begin{tabular}{|c|c|c|c|c|c|c|}
\hline Individual & Age (years) & Gender & Affected or not & $\begin{array}{c}\text { Age of onset/death } \\
\text { (years) }\end{array}$ & Phenotype & Genotype \\
\hline III-4 & 81 & M & $?$ & None & Asymptomatic & Duplication \\
\hline III-7 & 65 & M & Healthy & None & Asymptomatic & Normal \\
\hline III-9 & 63 & M & Affected & $50 /-$ & $\begin{array}{l}\text { Renal cell carcinoma, renal cyst, central } \\
\text { nervous system hemangioblastoma }\end{array}$ & Duplication \\
\hline III-11 & 57 & $\mathrm{~F}$ & Healthy & None & Asymptomatic & Normal \\
\hline III-14 & 56 & $\mathrm{~F}$ & Healthy & None & Asymptomatic & Normal \\
\hline III-16 & 52 & $\mathrm{~F}$ & Affected & $39 /-$ & $\begin{array}{l}\text { Central nervous system } \\
\text { hemangioblastoma, renal cyst }\end{array}$ & Duplication \\
\hline IV-3 & Unknown & $\mathrm{F}$ & Affected & $29 / 40$ & $\begin{array}{l}\text { Central nervous system } \\
\text { hemangioblastoma }\end{array}$ & Not detected \\
\hline IV-4 & 42 & M & Healthy & None & Asymptomatic & Normal \\
\hline IV-6 & 38 & $\mathrm{~F}$ & Affected & $33 /-$ & Glioblastoma & Duplication \\
\hline IV-7 & 40 & M & Healthy (spouse) & Unknown & Unknown & Normal \\
\hline IV-8 & Unknown & M & Affected & $27 / 28$ & Hemangioblastoma & Not detected \\
\hline IV-11 & 42 & M & Healthy & None & Asymptomatic & Normal \\
\hline IV-13 & 41 & $\mathrm{~F}$ & Healthy & None & Asymptomatic & Normal \\
\hline IV-15 & 34 & M & Healthy & None & Asymptomatic & Normal \\
\hline IV-21 & 40 & M & Healthy & None & Asymptomatic & Normal \\
\hline IV-23 & 36 & M & Affected & 33 & Renal cyst & Duplication \\
\hline IV-24 & Unknown & M & Affected & $21 / 30$ & Hemangioblastoma & Not detected \\
\hline IV-25 & 31 & $\mathrm{~F}$ & $?$ & None & Asymptomatic & Duplication \\
\hline IV-27 & 41 & M & Healthy & None & Asymptomatic & Normal \\
\hline IV-29 & 39 & $\mathrm{~F}$ & Healthy & None & Asymptomatic & Normal \\
\hline IV-31 & 34 & $\mathrm{~F}$ & Healthy & None & Asymptomatic & Normal \\
\hline IV-35 & 36 & M & Healthy & None & Asymptomatic & Normal \\
\hline IV-37 & 31 & $\mathrm{~F}$ & Healthy & None & Asymptomatic & Normal \\
\hline IV-39 & 26 & M & Healthy & None & Asymptomatic & Not detected \\
\hline IV-43 & 32 & M & Affected & 19 & Retinal hemangioblastomas & Duplication \\
\hline $\mathrm{V}-4$ & 19 & $\mathrm{~F}$ & Healthy & None & Asymptomatic & Normal \\
\hline $\mathrm{V}-5$ & 13 & M & Healthy & None & Asymptomatic & Normal \\
\hline V-6 & 16 & $\mathrm{~F}$ & $?$ & None & Asymptomatic & Duplication \\
\hline $\mathrm{V}-7$ & 11 & $\mathrm{~F}$ & $?$ & None & Asymptomatic & Duplication \\
\hline $\mathrm{V}-8$ & 8 & M & Healthy & None & Asymptomatic & Normal \\
\hline$V-11$ & 18 & $\mathrm{~F}$ & Healthy & None & Asymptomatic & Normal \\
\hline $\mathrm{V}-12$ & 10 & $\mathrm{~F}$ & Healthy & None & Asymptomatic & Normal \\
\hline$V-13$ & 7 & M & Healthy & None & Asymptomatic & Normal \\
\hline $\mathrm{V}-21$ & 10 & F & Healthy & None & Asymptomatic & Normal \\
\hline $\mathrm{V}-22$ & 5 & $\mathrm{~F}$ & Healthy & None & Asymptomatic & Normal \\
\hline$V-23$ & 3 & M & Healthy & None & Asymptomatic & Normal \\
\hline$V-24$ & 5 & M & $?$ & None & Asymptomatic & Duplication \\
\hline V-35 & 33 & M & Healthy & None & Asymptomatic & Normal \\
\hline
\end{tabular}

\section{Mutation detection}

We identified a novel 14-base pair duplication in exon 1 of $V H L$, which is predicted to result in a frameshift mutation and truncated pVHL30 (c.326-339dupTCCACAGCTACCGA, p.Gly114Serfs*50) and pVHL19 (c.167-180dupTCCACAGCTACCGA, p.Gly61Serfs*50) (Figure 3). The relative copy number of the $V H L$ of the proband was normal. Five affected individuals carried a heterozygous duplication; we also observed duplication in 5 asymptomatic relatives who were aged 81,31, 16, 11, and 5 (III-4, IV-25, V-6, V-7, and V-24), respectively. This variation was not present in the 70 normal control individuals. 


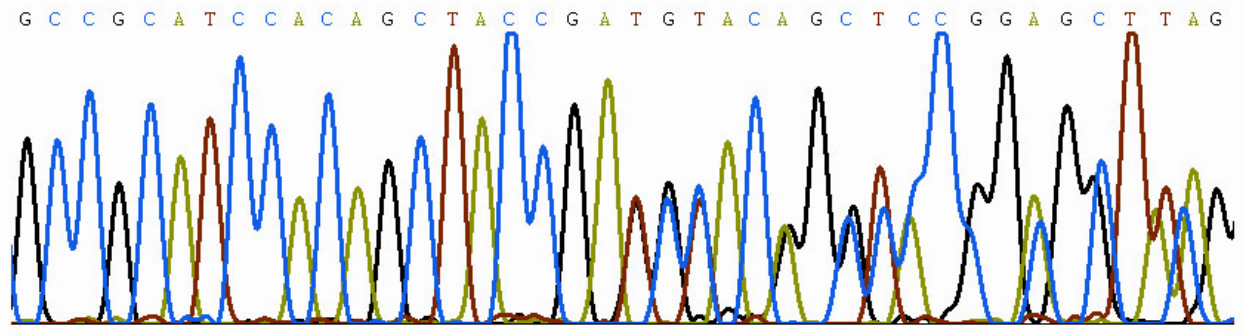

B

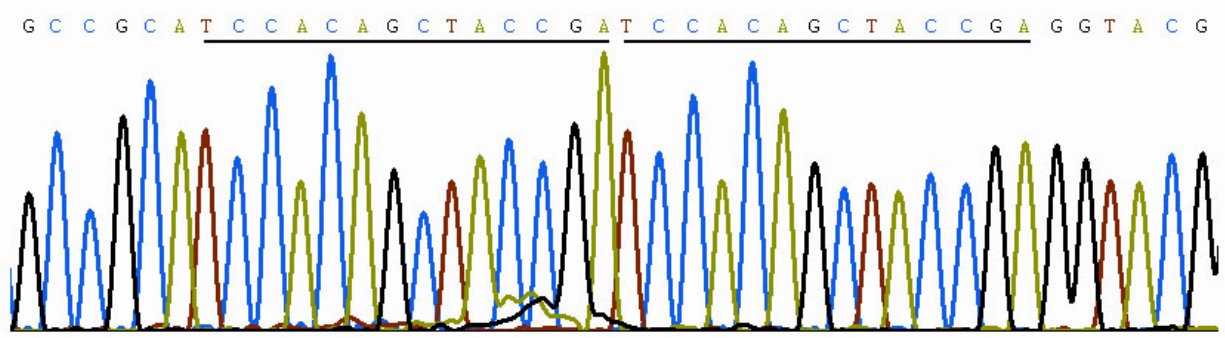

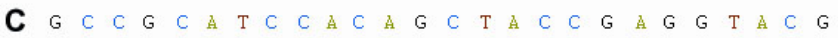

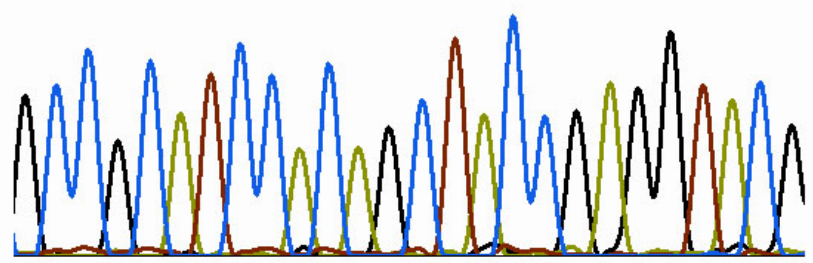

Figure 3. Mutation analysis of VHL. A. Genomic DNA sequencing of the proband. B. Clone sequencing of the mutant allele. C. Corresponding normal DNA sequence. The lines indicate the duplication region.

\section{DISCUSSION}

A wide spectrum of mutations can occur in DNA sequences, including missense mutations, nonsense mutations, microdeletions, microinsertions, splice mutations, and large deletions. According to previous studies, more than 500 mutations in $V H L$ have been reported, with most variants unique to individual families. Approximately $72 \%$ of $V H L$ variants are point variants or small deletions/insertions, while $28 \%$ are partial or complete deletions of the gene (Stolle et al., 1998; Hoebeeck et al., 2005; Banks et al., 2006).

Genotype-phenotype correlations have been observed in VHL disease, revealing associations between certain variants and the risk of either pheochromocytoma or renal cell carcinoma. Truncating mutations or missense mutations that are predicted to grossly disrupt folding of the VHL protein have been associated with VHL type 1 (low risk for pheochromocytoma), and some missense mutations appear to be correlated with a specific type 2 phenotype (high risk for pheochromocytoma) (Hes et al., 2000; Wong et al., 2007; McNeill et al., 2009). In this study, we reported a novel 14-base pair duplication mutation in a large 
family with VHL disease. This mutation was predicted to result in a frameshift and truncated pVHL30 and pVHL19, which causes a loss of the structural domain that interacts with elongin $\mathrm{B}$ and C. Sixty percent of the living patients had hemangioblastomas and renal cysts and 1 developed renal cell carcinoma. No affected individuals developed pheochromocytoma and their phenotypes were consistent with type 1 . However, the relationship between a germline mutation in the $V H L$ gene and the manifestation (age of onset and type) of VHL-related tumors is complex, and the VHL phenotype observed varies among families, although even among members of the same family, some genetic ('modifier' genes) and/or environmental factors may be involved in clinical symptoms. There is typically nearly complete penetrance by the age of 70 years (Maher et al., 1991); however, this family included an 81-year-old obligate heterozygote (both parent and children affected) who had no symptoms of VHL disease. To the best of our knowledge, this is the oldest asymptomatic relative identified to date; however, the individual refused to undergo screening to detect asymptomatic lesions.

Advances in understanding the genetic basis of VHL disease have facilitated diagnosis and provided insight into the biology of this disease. Molecular genetic testing for early identification of at-risk family members improves diagnostic certainty and reduces the need for costly surveillance, and eases the psychological burden of family members who have not inherited the disease-causing mutation. Surveillance of affected and asymptomatic gene carriers can reduce morbidity and mortality. In this family, we identify 5 affected and 5 asymptomatic carriers, all requiring regular clinical monitoring such as ophthalmologic screening, blood pressure measurement, audiologic evaluation, abdominal ultrasound examination, cranial computerized tomography scan, or magnetic resonance imaging.

In summary, we identified a novel small duplication resulting in severely truncated pVHL30 (p.Gly114Serfs*50) and pVHL19 (p.Gly61Serfs*50) in a large family with VHL disease. All patients and asymptomatic gene carriers require regular clinical monitoring throughout life to reduce mortality.

\section{ACKNOWLEDGMENTS}

Research supported by the National Natural Science Foundation of China (\#81000253) and the Specialized Research Fund for the Doctoral Program of Higher Education of China (\#20102104120024). We thank the patients and their family for participating in this study. We give special thanks to the proband who gave permission to use his photograph in this publication.

\section{REFERENCES}

Banks RE, Tirukonda P, Taylor C, Hornigold N, et al. (2006). Genetic and epigenetic analysis of von Hippel-Lindau (VHL) gene alterations and relationship with clinical variables in sporadic renal cancer. Cancer Res. 66: 2000-2011.

Hes F, Zewald R, Peeters T, Sijmons R, et al. (2000). Genotype-phenotype correlations in families with deletions in the von Hippel-Lindau (VHL) gene. Hum. Genet. 106: 425-431.

Hes FJ, Höppener JW, Luijt RB and Lips CJ (2005). Von hippel-lindau disease. Hered. Cancer Clin. Pract. 3: 171-178.

Hoebeeck J, van der Luijt R, Poppe B, De Smet E, et al. (2005). Rapid detection of VHL exon deletions using real-time quantitative PCR. Lab. Invest. 85: 24-33.

Kaelin WG Jr and Maher ER. (1998). The VHL tumour-suppressor gene paradigm. Trends Genet. 14: 423-426.

Maher ER, Iselius L, Yates JR, Littler M, et al. (1991). Von Hippel-Lindau disease: a genetic study. J. Med. Genet. 28: 443-447.

Maher ER, Neumann HP and Richard S (2011). von Hippel-Lindau disease: a clinical and scientific review. Eur. J. Hum. Genet. 19: 617-623. 
McNeill A, Rattenberry E, Barber R, Killick P, et al. (2009). Genotype-phenotype correlations in VHL exon deletions. Am. J. Med. Genet. A 149A: 2147-2151.

Schoenfeld A, Davidowitz EJ and Burk RD (1998). A second major native von Hippel-Lindau gene product, initiated from an internal translation start site, functions as a tumor suppressor. Proc. Natl. Acad. Sci. U. S. A. 95: 8817-8822.

Stebbins CE, Kaelin WG Jr and Pavletich NP (1999). Structure of the VHL-ElonginC-ElonginB complex: implications for VHL tumor suppressor function. Science 284: 455-461.

Stolle C, Glenn G, Zbar B, Humphrey JS, et al. (1998). Improved detection of germline mutations in the von HippelLindau disease tumor suppressor gene. Hum. Mutat. 12: 417-423.

Wong WT, Agrón E, Coleman HR, Reed GF, et al. (2007). Genotype-phenotype correlation in von Hippel-Lindau disease with retinal angiomatosis. Arch. Ophthalmol. 125: 239-245. 